\title{
Providing and Optimizing a Robotic Construction Plan for Rescue Operations
}

\author{
Hadi Ardiny, Stefan Witwicki, and Francesco Mondada ${ }^{1}$
}

\begin{abstract}
After a terrible disaster such as an earthquake or a nuclear accident, finding victims and isolating them from hazards are usually the first priorities for rescuers. As the security of rescuers and the stabilization of the environment are critical components of the first rescue phase, we assume that robots could be used to secure the environment by performing construction tasks, to stabilize large structures, and/or protect the victims. In this paper we suggest an approach consisting of using mobile robots to construct protective walls on a site affected by a nuclear disaster. Protective walls can help to block radiation from toxic sources and protect both victims and rescuers. On the other hand, the robot's vulnerability to radiation restricts its freedom of movements into unsafe regions. Therefore, building protective walls needs a plan (construction plan) that involves three competing objectives: victim safety, rescuer safety, and robot safety. Weighting these factors is a societal choice, is not trivial, and impacts the whole system.

In this paper, we provide and optimize the construction plan using a genetic algorithm based on three objectives. We analyze the construction plan performance with respect to execution time. We also analyze the trade-offs involved between these competing objectives in different environments with ranging physical complexity (e.g., a number of victims or sources).
\end{abstract}

\section{INTRODUCTION}

\section{A. Motivation}

With recent progress in the field of robotics, the potential for rescue robots to help society is growing. Human operators can employ rescue robots as assistants in hazardous and restricted environments to access trapped victims. A terrible disaster such as an earthquake or a nuclear accident destroys city infrastructures and potentially hurts a number of people. The goal of the search and rescue operation is to find and isolate victims from the hazards in the shortest amount of time. Obviously, search and rescue operations must avoid creating additional risks to rescuers and victims (save lives). For that reason, the rescue team must act quickly and securely to get information and to save victims within the shortest time in a potentially vast disaster area. These victims might be threatened with several kinds of dangers such as falling walls or exposure to toxic radiation. Accordingly, one of the rescue services that mobile robots can contribute is to build particular structures to stabilize the environment, especially where human operators cannot easily or safely reach.

\footnotetext{
${ }^{1}$ Hadi Ardiny, Stefan Witwicki, and Francesco Mondada are with Robotic systems Laboratory, École Polytechnique Fédérale de Lausanne, Lausanne, Switzerland hadi.ardinydepfl.ch, stefan.witwickidepfl.ch, francesco.mondada@epfl.ch
}

The long-term goal of this research is to enable mobile robots to autonomously secure hazardous environments using various methods of construction. In this work, an indoor environment is assumed to have toxic radiation after a radioactive disaster, such as a nuclear laboratory incident. The idea is to decrease victims' exposure to toxic radiation by building protective walls in the environment. We aim to identify where robots can build protective walls efficiently. In fact, we deal with two questions: where to build walls and in what order to build. Answering these questions provides a construction plan which directs robots for an efficient rescue operation. More specifically, in this work, the construction plan is a 2D map of protective walls which comprises protective walls shapes, their positions, and directions.

Radiation can damage tissue and organs depending on the effective radiation absorbed by the body. Moreover, some electronic components such as semiconductors are very sensitive to radiation. Therefore, a construction plan directs robots to build walls that satisfy the following objectives: victim safety, rescuer safety, and robot safety. In other words, minimizing cumulative exposure to radiation for victims, rescuers and robots is the goal of the construction plan. We thereby frame the construction planning problem as an optimization of three competing objectives.

Towards solving this nontrivial optimization, we develop a genetic algorithm (GA), which is an evolutionary algorithm based on iterative randomized search. GAs have the potential to find good approximate solutions for problems which are not able to be solved with standard optimization methods. Moreover, they can straightforwardly be applied to problems in which the objective function is non-differentiable, complex, discontinue, or highly non-linear. A strength of the GA is the use of (1) stochastic search to well cover the solution space, combined with (2) meta-heuristics that focus in on promising regions of the space. Nevertheless, GAs do not guarantee that an optimal solution will be found; we may consider their application as an approximation method. In this work, we thereby benefit from the GA to characterize our nontrivial problem and find a solution close to the best one. Our method can subsequently serve as a benchmark to compare the performance of other construction plan methods.

Providing and optimizing the construction plan using a GA will be the first contribution of this research. The second contribution is to present the trade-offs involved between the three objectives. We hope that our analysis can inform societal choices about future adoption and operation of autonomous 
mobile robots in rescue scenarios. Finally, we analyze how the objectives are sensitive to the effects of physical complexity (e.g., increasing the number of victims or sources).

Section II provides a description of the problem and assumptions. Section III explains our proposed GA in more detail. In Section IV, we discuss the results. Finally, we conclude the research and propose future work.

\section{B. Related work}

We primarily review studies that develop algorithmic construction plans, although most previous work on autonomous construction has focused on other aspects. Previous research on autonomous construction can be classified in terms of the construction plan as follows: (1) entirely pre-specified construction plan; (2) simple construction plan (e.g., straight line) with the influence of environmental features; and (3) construction plan influenced by environmental features without any pre-specified shape to satisfy abstract goals.

Some researchers present the construction of specific structures in which the shape is fully pre-specified and requested by a user [1]. Werfel [2] has demonstrated a simple simulation in which a group of robots tries to rearrange blocks into a 2D shape based on a high level geometric shape. This shape is given by the user and the robots try to build the structure. Moreover, Werfel et al. [3] present a 3D collective construction in which the system receives a high-level representation of a desired structure and translates it into some rules based on collective robotic behaviors. Similarly, a team of quadrotors has been controlled to build 3D structures using blocks or rods based on relatively complicated pre-specified shapes (e.g., block tower, truss) [4].

Some construction algorithms take input from both prespecified shapes and the influence of environmental features. Melhuish et al. [5] report a simple 2D wall building by groups of robots over linear strips pre-placed on the floor. The robots used linear strips as markers by depositing material at a certain distance from them. In [6], an organizer robot coordinated the building activities of robots by generating a light-field pattern that varied in space and time. This template was employed by robots to build a loose linear wall according to the light-field pattern. Soleymani et al. [7] have developed a construction system in which robots build a protective barrier to fill a rectangular area.

Conversely, some research presents construction plans that may not need to be fully specified in advance, so they can allow a certain amount of dynamic flexibility during the construction process. Napp and Nagpal [8] propose algorithms with amorphous materials that enable robots to build arbitrary shapes. The idea has been described for an adaptive ramp building by which robots fill a valley and provide access to other sides. A compilation procedure efficiently encodes a ramp structure to arbitrary shapes with a relatively small number of markers. This method allows robots to build ramps by locally reacting to markers. Werfel et al. [9] have proposed an adaptive structures algorithm. In this case, no predefined shape is considered and environmental features (e.g., chemical spill shape) will determine it. In other words, a team of robots would be tasked to build a protective barrier around a hazardous chemical spill with a given thickness. They just address a shape, although these construction plans are flexible in construction. In fact, they do not comprise other issues which can be important in construction for rescue operations such as order and direction of each structure.

In conclusion, these three classifications span the range from entirely pre-specified construction plans to those whose shapes are determined by the influence of environmental features. In this paper, we address a new construction plan influenced by environmental features. In addition to shape of construction, it comprises more information to build the walls to efficiently reduce cumulative exposure for important elements such as victim. For instance, the order and the direction of each protective wall that affects the cumulative exposure to radiation for the victims are addressed. Moreover, another novelty of this research is to optimize the construction plan based on multiple objectives; thus, we also study the trade-offs involved between the objectives. In the next section, we discuss the objectives and their roles in more detail.

\section{Problem Statement}

\section{A. Description}

We consider rescuers who want to employ robots for rescue in an environment which has toxic radiation after a disaster, such as after a nuclear laboratory incident. The existence of toxic sources makes the rescue operation dangerous and restrictive to rescuers. In this situation, a spontaneous rescue effort might result in compounded problems. Therefore, this disaster area should be scanned and secured to avoid the problems associated with an unsafe environment. Robots can be useful in rescue operations by gathering facts about the disaster area and then reducing the risks by building protective walls, thereby blocking radiation from toxic sources for both victims and rescuers. Building these walls provides safe paths for victims and rescuers; rescuers can safely access victims to retrieve them and administer treatment.

According to time limits and safety issues to save lives in rescue operations, a plan is necessary to direct construction tasks. We compute a construction plan which directs robots to build walls that seek to balance three objectives (low radiation exposure for victims, rescuers and robots). Victims have to be protected as the first priority because cumulative toxic radiation exposure can seriously damage tissue and organs. Rescuers need safe paths to perform rescue tasks, although providing completely safe paths need more time and robotic effort. On the other hand, some electronic components such as semiconductors are very sensitive to radiation and it might cause the robots' behaviors to fail. Therefore, the robot safety objective tries to decrease cumulative radiation exposure on robots (and hence the robots' presence) in dangerous regions 
for construction. As a result, we deal with competing objectives and the weights have to be chosen based on the applications. For instance, if a robot is shielded against the radiation, or if we make sure the radiation intensity does not make any trouble for the robot according to the sensitivity test were performed before its deployment [10], the related weight could be lower than the others or even set to zero.

In this paper, we seek to compute and optimize the construction plan based on three competing objectives using the GA. The encoded map is used by the GA as an input. In Section III, we describe the proposed GA in more detail.

\section{B. Assumptions}

We assume a rectangular flat environment which is surrounded by walls. It may also have internal walls. Victims and safe regions are randomly placed on the environment for each experiment. We assume that toxic radiation sources emit rays from their centres, so they can be seen as points. Furthermore, they have the same intensity, and emit linear and continuous rays.

According to the inverse square law for radiation fields, the radiation dose of a toxic source is inversely proportional to the square of the distance from the toxic source. As a result, the radiation dose of each point is given by adding up the decreased dose of radiation sources[11, 12], as can be seen in Eq. (1)

$$
g(x, y)=\sum_{i=1}^{N_{r}} \frac{I_{i}}{\sqrt{\left(x-X_{r_{i}}\right)^{2}+\left(y-Y_{r_{i}}\right)^{2}}}
$$

\section{Where}

$g(x, y)$ gives the dose of the cell $(\mathrm{x}, \mathrm{y})$

$I_{i}$ is the intensity of the radiation source for the $i$ th source

$N_{r}$ is the number of radiation sources

$X_{r_{i}}, Y_{r_{i}}$ is the position of the radiation source for the $i$ th source

In Eq. 1, we compute cell dosages for each radiation source that is connected to the cell by an imaginary straight line without any intersection with walls or obstacles. Victims are modelled as filled rectangles and they are of the same size and shape. We suppose the use of a wheeled autonomous robot which is equipped with a 3-DOF robotic arm and a gripper. The robot picks up and deposits a block made of a particular material that blocks the radiation. The doors are connected to safe regions, as illustrated in Fig. 1. Moreover, various elements (victim, toxic source, and doors) are scattered randomly with unknown positions.

The robotic construction tasks consist of four steps: move towards the safe region, pick up a block from the depot, move toward the deposition place, and release the block, as shown in Fig. 1. We assume that robots spend significantly more time depositing blocks than moving them; thus, we neglect exposure to radiation for the robot during movement. their exposure is also zero when the robots pick up the blocks because depots are placed in safe areas. Therefore, cumulative

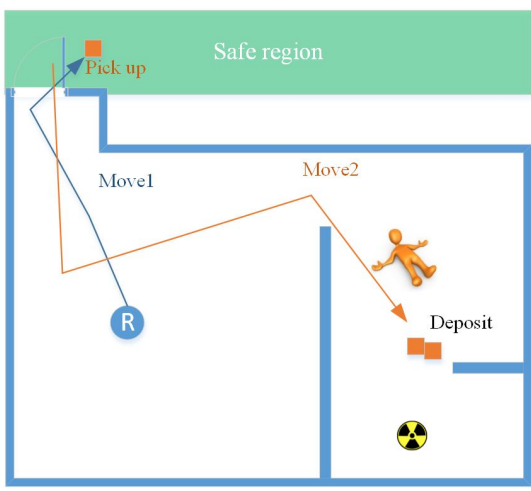

Fig. 1. A schematic of an environment with four robotic activities: 1. moves toward the safe region; 2 . picks up the block; 3 . moves toward to the construction place; 4 . deposits the block

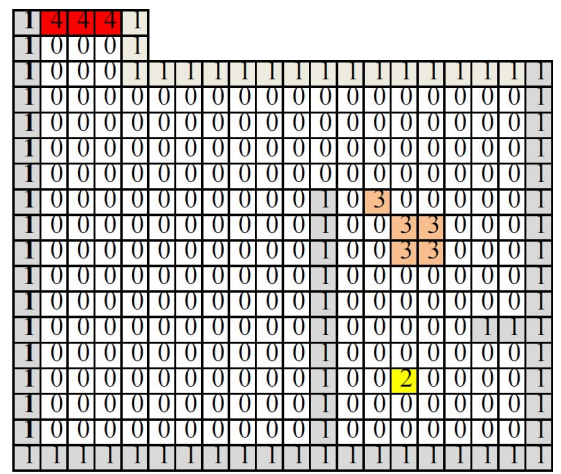

Fig. 2. A representation of an encoded environment which is shown in Fig. 1 as a grid-cell; 0 shows free cells and 1,2,3, and 4 show occupied cells with the walls (or obstacles), toxic sources, victims, and doors respectively. We use the encoded environment for the GA as an input to compute the construction plan.

robot exposure is calculated based on the depositing block task.

Without loss of generality, we assume that construction speed (including all four tasks) is 1 block per time units (e.g., 1 block/minute) and rescuer speed is also equal to 1 cell per time unit (we envision the environment composed of gridcells, so the speeds are described based on cells per time unit). Of course, if we change the construction speed, the range of exposure for victims and robots will proportionally change. For instance, the presence of obstacles makes the robot slower to build the protective walls; in this case the construction speed should decrease. Similarly, the range of rescue exposure is proportional to the speed of the rescuer. This means that the range of rescue exposure will decrease with an increase of rescuer speed.

Calculating rescuer and robot exposure depends on prespecified paths between victims and safe areas. In fact, the rescuers need a pre-specified path to access victims, so we need to determine the shortest path between victims and the nearest safe region. Fortunately, in past decades, many path 
finding methods have been developed [13]. Accordingly, we apply the wave-front path finding method, which is commonly used in grid maps to find the shortest path.

\section{Genetic Algorithm Approach}

Genetic algorithms (GAs) are global search and optimization techniques taking inspiration from evolutionary theory. We view the GA is also a powerful tool to optimize non-trivial problems such as we face here. Building on several applications of GA in the field of robotics (e.g., robot navigation) [14], we encode the map as a grid whose cells encode certain features. As depicted in Fig. 2, cells' values indicate which elements (wall or obstacle, victim, toxic source, free) occupy the corresponding cells.

Encoding the protective walls is another issue. Each protective wall is a segment, which is defined by its start and end positions. Both the start and end positions are in an array of integers that make up a chromosome. Inversely, each chromosome contains genes which are start $\left(x_{s_{j}}, y_{s_{j}}\right)$ and end positions $\left(x_{e_{j}}, y_{e_{j}}\right)$. The chromosome $k$ is of the form:

$$
\begin{gathered}
\text { chromosome }_{k}=\left[x_{s_{1}}, y_{s_{1}}, x_{e_{1}}, y_{e_{1}}, x_{s_{2}}, y_{s_{2}}, x_{e_{2}}, y_{e_{2}},\right. \\
\left.\ldots, x_{s_{n}}, y_{s_{n}}, x_{e_{n}}, y_{e_{n}}\right]
\end{gathered}
$$

where

$$
x_{s_{j}}, y_{s_{j}}, x_{e_{j}}, y_{e_{j}} \in \mathbb{N}, \forall j \in\{1, \ldots, n\}
$$

The chromosome $k$ consists of $n$ genes, while each segment (or protective wall) has been defined by four genes of this chromosome.

After each evaluation, the GA removes the worst solutions, and in place of these, breeds new ones from the best design solutions. Each new solution must be evaluated by a fitness function to indicate rank of each solution is (solutions are sorted from best to worst). A fitness function generates a fitness value from the corresponding chromosome. The fitness function which comprises the three discussed objectives is defined as:

$$
\begin{aligned}
\text { fitness } & =\left(1+w_{1} \times V E+w_{2} \times R E\right. \\
& \left.+w_{3} \times R E S E\right)^{-1} \\
& \left(w_{1}+w_{2}+w_{3}=1\right)
\end{aligned}
$$

where:

- $\left(w_{1}, w_{2}, w_{3}\right)$ are corresponding weights,

- $V E$ is the cumulative dose of radiation (e.g., Gray ${ }^{1}$ units) absorbed by victims during the whole process,

- $R E$ is the that absorbed by robots during construction,

- RESE is that absorbed by rescuers.

Eq. (1) gives the exposure rate (Gy/time units) for each position, and the integral of exposure rates over time are cumulative exposure of elements ( $V E, R E, R E S E)$. Moreover,

\footnotetext{
${ }^{1}$ The gray (Gy) is defined as the absorption of one joule of radiation energy by one kilogram of matter in SI
}

the exposure terms in the fitness function are inversely proportional to the discussed objectives; for example, victim safety objective is equal to victim_exposure ${ }^{-1}$.

The genetic operations are as follows: algorithm selection, crossover, and mutation. In algorithm selection, the solutions (population) are ranked from highest fitness to lowest fitness. Then, only a fraction of the population is selected to survive, while the rest are removed. In the next step, we assign probability for every chromosome to breed the next generation according to a certain probability. The probability assigned to a chromosome is proportional to its fitness. By the crossover operator, two chromosomes are selected from survival chromosomes to produce two new offspring based on a random crossover point. By mutation operators, we replace a certain percentage of the genes (integer numbers) in the list of chromosome with new random integer numbers.

A GA maintains a population of candidate solutions. The initial population is generated at random. The length of the chromosome is assumed to be variable. The initial number of genes is eight times greater than the number of toxic sources, because in a worst-case scenario of the possible configuration of elements on the environment, the toxic sources can be isolated by a near shape with four lines (e.g., a square shape). A termination condition stops the GA when the number of generations reaches a threshold.

\section{EXPERIMENTAL RESULTS}

\section{A. Performance of GA for selected weights}

Before starting the primary experiments, we studied the performance of the GA. We assumed an environment with a fixed arrangement of elements and repeated trials 10 times for each point illustrated in Fig. 3. As described by Eq. (3), the fitness function consists of three terms and corresponding weights. By changing weights, we can manipulate the fitness function to direct the construction plan to satisfy the objectives. For instance, point $\mathbf{P}$ in Fig. 3 shows $0.25,0.5$, and 0.25 for $w_{1}$, $w_{2}$, and $w_{3}$, respectively. The results for performance of the GA with a fixed arrangement of elements shows that the GA converges to almost the same fitness value for each point (the average relative deviation from the mean for fitness value is equal to $5 \%$ ).

We considered a type of environment after a disaster with two different configurations of elements on the environment: the first one is an environment with a victim, a toxic source, and two doors as a simple environment (Fig. 4a); second one is an environment with two victims, two radiation sources, and two doors as a complex environment (Fig. 4b). Our results thereby focus on only a small subset of scenarios so as to enable a rigorous and systematic evaluation across several key parameters.

Ten trials were carried out for each point illustrated in Fig. 3 , while elements were scattered randomly in each trial. We demonstrate the detailed results of one selected trial with the following weights: $w_{1}=0.3, w_{2}=0.35, w_{3}=0.35$. In Fig. 4 


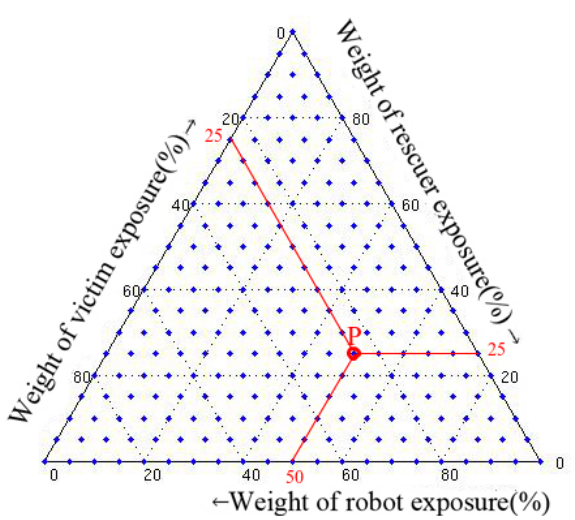

Fig. 3. Weight balancing triangle; the blue points reflect used weights for the experiments, point $\mathbf{P}$ shows a sample for the corresponding weights of each point. For instance, point $\mathbf{P}$ shows $0.25,0.5$, and 0.25 for $w_{1}, w_{2}$, and $w_{3}$, respectively.

we show two construction plans from the GA. The protective walls are shown in green in the environment. The paths, sources, victims and entry doors to the safe area (or depot) are illustrated by blue, yellow, red, and brown, respectively. For this sample, the protective walls don't completely protect the path. If a construction plan considers longer walls, cumulative robot exposure will increase while victims are completely protected. This confirms that the two objectives are competing.

Fig. 5a illustrates the performance of the GA for the construction plan. It shows that the proposed GA quickly reaches a steady fitness.

The graph which is shown in Fig. 5b depicts the exposure to radiation for victims versus time in the simple and complex environments. It starts when robots deposit the first block. As can be seen, the construction plan tries to decrease the cumulative exposure of the victims (integral of victims exposure graph) quickly. Because the victims are exposed to radiation, the robots must try to protect them quickly. Otherwise, cumulative exposure will increase if the robots act slowly or perform unnecessary tasks. Also, a comparison of the two configurations (simple, complex) shows that cumulative exposure tends to increase with an increase in the complexity. The graph shown in Fig. 5c illustrates the exposure to radiation for robots versus the time when robots start to build protective walls. It shows that the radiation exposure of robots declines versus time because constructed protective walls prevent further exposure. On the other hand, these graphs are not smooth because robots deposit blocks in several places with differing radiation intensity. As a result, a construction plan tends to decrease both victim exposure and robot exposure.

Fig. 6 shows that the execution time of the GA raises with the complexity. We increase complexity by the number of victims and sources at the same environment with random arrangement of the elements. The execution time also depends

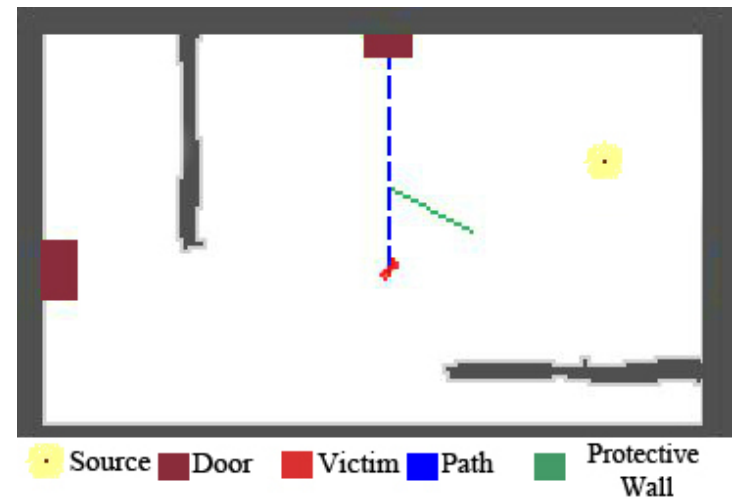

(a) Dangerous environment with a victim and a toxic source (simple environment)

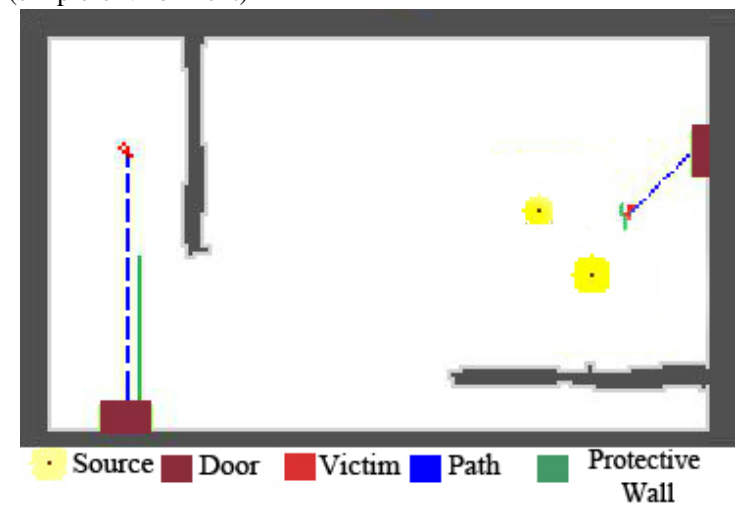

(b) Dangerous environment with two victims and two toxic sources (complex environment)

Fig. 4. Two construction plans (wall shape shown) computed by the GA; the green color depicts protective walls, the blue, yellow, red, and brown colors depict the paths, toxic sources, victims, and entry door to the safe areas, respectively. We predetermine shortest paths between doors and victims because the construction plan is made based on these paths.

on the environment's dimensions. ${ }^{2}$

\section{B. Objectives trade-off}

In this section, we study the trade-offs involved between competing objectives (victim safety, robot safety, and rescuer safety). As described by Eq. (3), the fitness function consists of three terms and corresponding weights. By changing weights, we can manipulate the fitness function to direct the construction plan to satisfy the objectives. For instance, point $\mathbf{P}$ in Fig. 3 shows $0.25,0.5$, and 0.25 for $w_{1}, w_{2}$, and $w_{3}$, respectively, so this construction plan intends to keep robots safer rather than victims and rescuers.

Therefore, the trade-off of objectives is done by changing the corresponding weights. To represent objectives' trade-off, we create a particular triangle to plot all possible weights. Each point inside of this triangle gives us the value of the corresponding weights. Each edge represents a systematic variation of the weight between 0 and 1 . It is divided into 20

\footnotetext{
${ }^{2}$ A real-world disaster environment could be substantially larger than that used in our experiments, and evaluating the efficiency of construction planning in such environments is a topic of future work.
} 


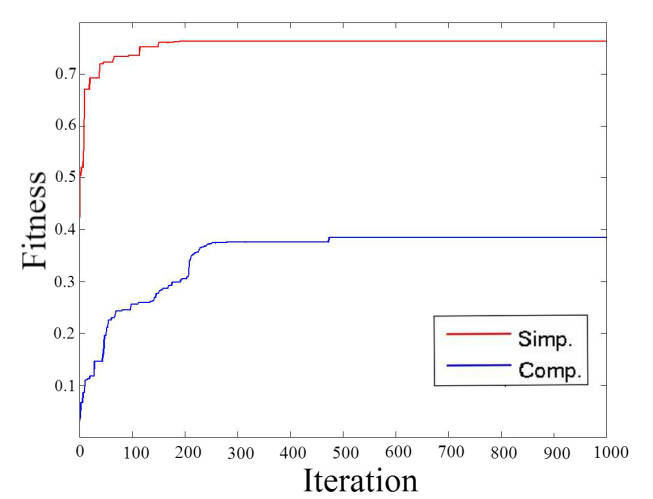

(a) Fitness value versus iteration to show performance of the GA for the selected situation.

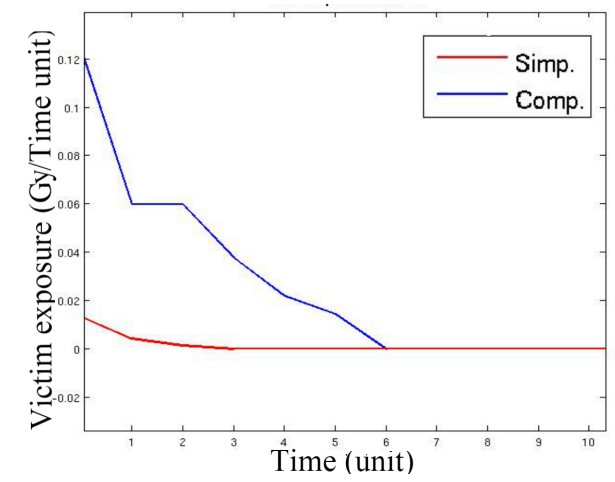

(b) Victim radiation exposure versus time during the execution of the building plan.

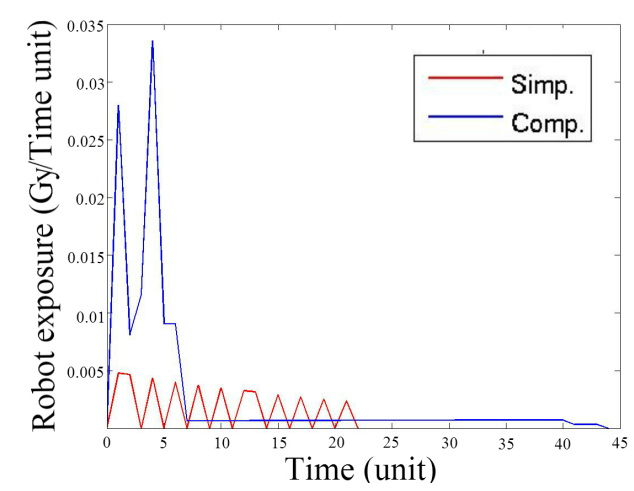

(c) Robot radiation exposure versus time during the execution of the building plan.

Fig. 5. The corresponding graphs of the construction plan performance for a set of arbitrary weights $\left(w_{1}=0.3, w_{2}=0.35, w_{3}=0.35\right)$ for two selected situations; the red color shows the simple environment and the blue color shows the complex environment.

sections, and then this triangle provides 231 points of possible weights, as can be seen in Fig. 3 .

The result of weight balancing is shown in Fig. 7. For each point, the trials were carried out 10 times with a random arrangement of the elements (victims, doors, sources). Likewise, we assume two configurations, simple (one victim, two doors, one toxic source) and complex (two victims, two doors, two toxic sources). The graphs show the average values

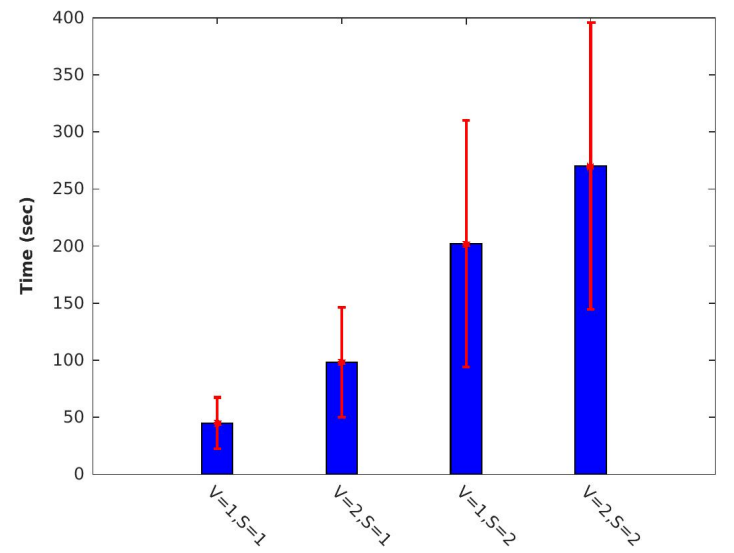

Fig. 6. The execution time of the GA versus the complexity. $V$ and $S$ indicate the number of victims and sources respectively.

of 10 trials for each point. Victim, robot and rescuer exposure are cumulative exposure values.

The results report that victim exposure is more robust to variations in the weight, except when the related weight is close to zero. In contrast, rescuer exposure is more sensitive at lower values of rescuer weight because most protective walls are devoted to covering the linking paths.

Moreover, rescue exposure does not have a uniform dispersion. For instance, as you can see in the average rescue exposure for the complex configuration in Fig. 7-C2, if the rescue weight equals 0.2 and we decrease the robot weight (or increase victim weight), the rescuer exposure decreases. In other words, robots have to limit their presence in the environment; thus, they decrease the building of protective walls. This means that these objectives are correlated.

Furthermore, a comparison of simple and complex configurations shows that exposure dispersion patterns stay the same. It is an interesting point because we can potentially define a particular region inside of the triangle to guarantee the performance of the construction plan. The same imaginary area is assumed inside the victim, rescuer, and robot exposures. We call this a reliable area which provides users with a set of reliable weights. These weights should guarantee a reliable construction plan, while the three exposure values are less than 20 percent of the maximum value. Therefore, the reliable area would keep its boundary versus increasing complexity because the exposure dispersion patterns stay the same.

The values of the same point inside of the corresponding triangles are not constant versus increasing complexity. The range value of victim exposure has grown tremendously. Maximum victim exposure increased seven times, while the number of victims and number of sources have been multiplied by two. However, maximum robot exposure was multiplied by 4 and maximum rescuer exposure increased twice in respect to increasing complexity. 


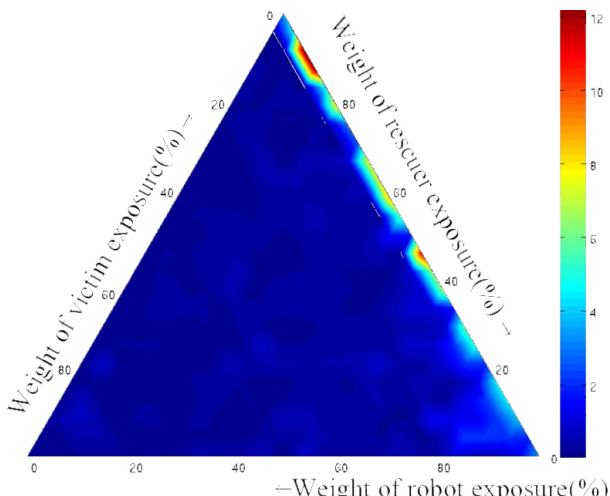

-Weight of robot exposure $(\%)$

Al. Average victim exposure

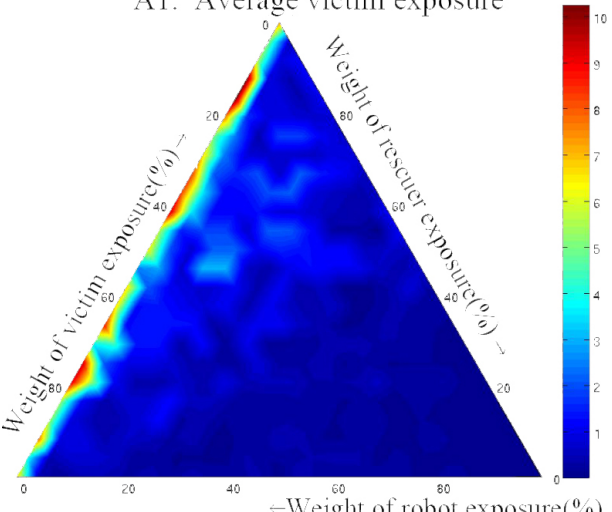

B1. Average robot exposure

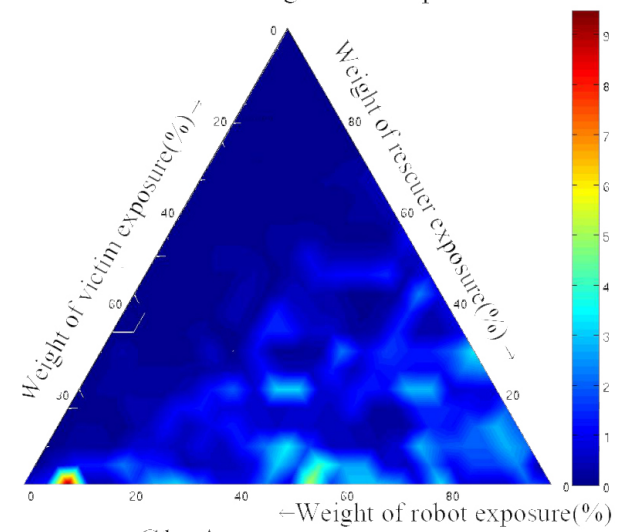

Cl. Average rescuer exposure

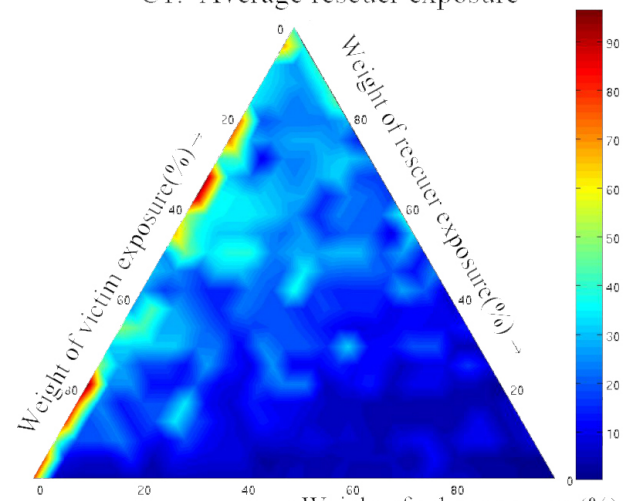

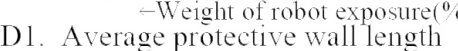

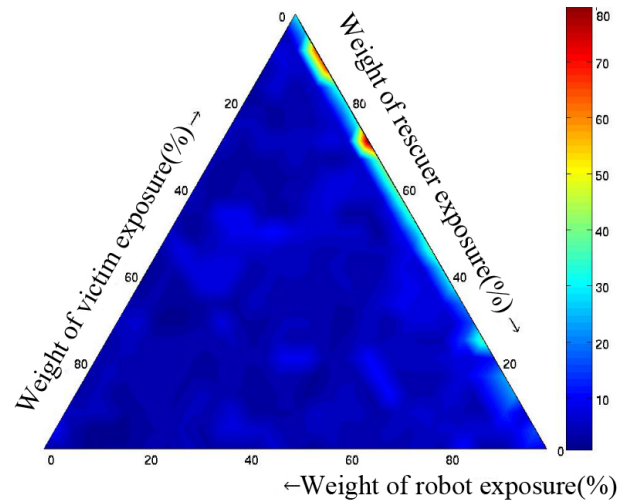

A2. Average victim exposure

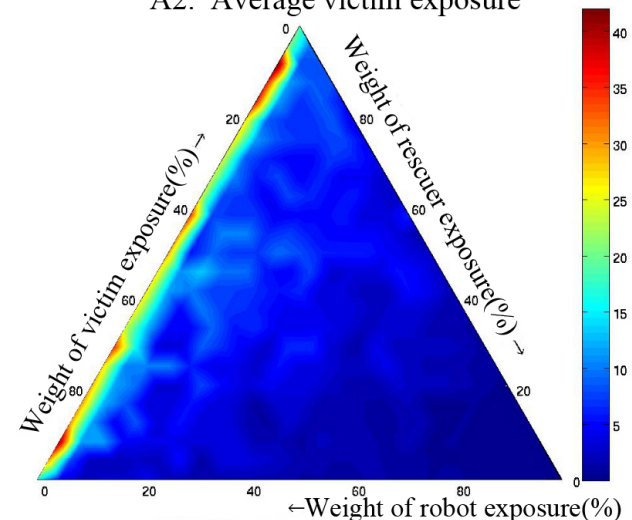

B2. Average robot exposure

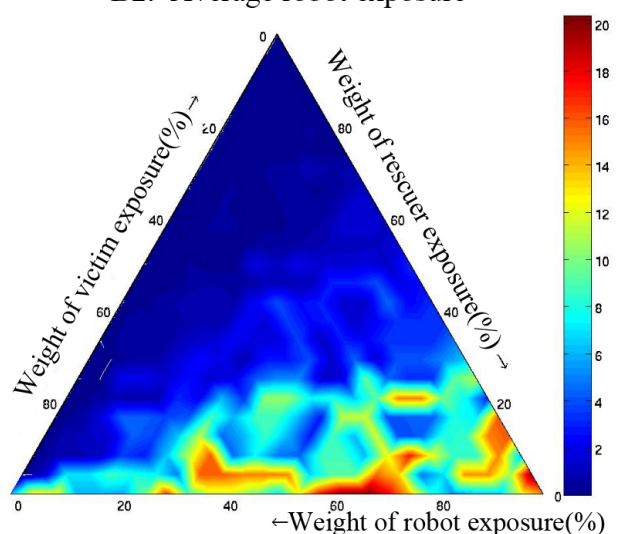

C2. Average rescuer exposure

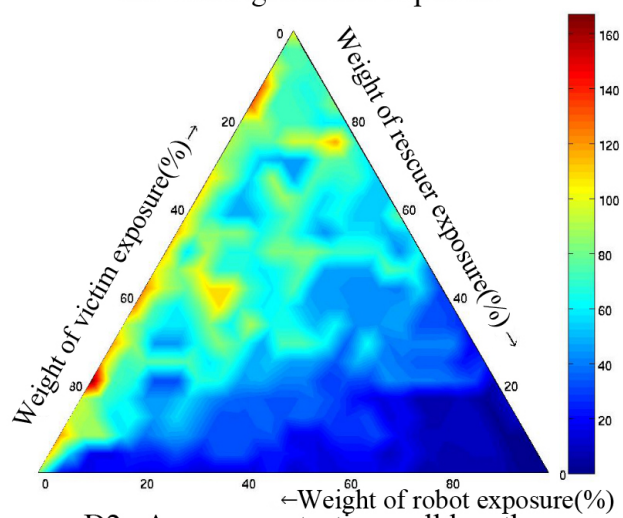

D2. Average protective wall length

Fig. 7. Objectives' trade-off results. The left graphs belong to simple environment configuration and the right graphs belong to complex environment configuration. 
In addition to the victims, doors, and rescuers exposures, the protective wall lengths are shown in Fig. 7-D. The duration of construction is a vital factor for the rescue operation because victims have to be accessible in a short time by rescuer. We assumed a constant speed of construction; therefore, the time of construction is proportional to the protective wall lengths. Fig. 7-D shows the total protective wall lengths. Its dispersion pattern has been changed by increasing complexity. We also expect that the growth rate of the walls is equal to four since we increase the complexity, but the result demonstrates that it increases less than twice. This shows that there is some overlap between the protective walls, so some parts of the protective walls are able to secure more of the environment than we expected.

\section{CONCLUSION}

In this paper, we present a method to compute a construction plan for mobile robots that would perform construction of protective walls in a nuclear disaster scenario. This construction plan takes into account three competing objectives: victim safety, robot safety, and rescuer safety. By studying the trade-offs between these objectives, we have shown the impact of the chosen weighting approach, highlighting for instance some correlations between victim and robot safety. We also determined a set of weights ensuring 80 percent for all three objectives. The experiments presented in this paper demonstrate that this set of weights is not impacted by a small variation of complexity. Finally, we analyzed the impact of complexity (e.g., the number of victims or sources) on the objectives, showing that rescue exposure is the first and most significantly affected factor when the complexity increases. According to time limits and safety issues to save lives in disaster areas, we hope that this research opens new approaches to enable rescue teams for faster and safer rescue operations.

In future work, we would like to move to the execution of these plans on simulated and real robots (a topic we have already begun to investigate [15]). In addition, we are looking for less time consuming methods, in respect to GAs, for the creation of the construction plan.

\section{ACKNOWLEDGMENTS}

This work was partially supported by the Swiss National Center of Competence in Research "Robotics".

\section{REFERENCES}

[1] S. Wismer, G. Hitz, M. Bonani, A. Gribovskiy, and S. Magnenat, "Autonomous construction of a roofed structure: Synthesizing planning and stigmergy on a mobile robot," in Intelligent Robots and Systems (IROS), 2012 IEEE/RSJ International Conference on, Oct 2012, pp. 5436-5437.

[2] J. Werfel, "Building blocks for multi-robot construction," in 7th International Symposium on Distributed Autonomous Robotic Systems, 2004.
[3] J. Werfel, K. Petersen, and R. Nagpal, "Distributed multirobot algorithms for the termes $3 \mathrm{~d}$ collective construction system," in Proceedings of Robotics: Science and Systems VII, 2011.

[4] J. Willmann, F. Augugliaro, T. Cadalbert, R. D'Andrea, F. Gramazio, and M. Kohler, "Aerial Robotic Construction Towards a New Field of Architectural Research," International Journal of Architectural Computing, vol. 10, pp. 439-460, 2012.

[5] C. Melhuish, J. Welsby, and C. Edwards, "Using templates for defensive wall building with autonomous mobile ant-like robots," in Proceedings of Towards Intelligent Autonomous Mobile Robot. Morgan Kaufmann Publishers, 1999, pp. 1495-1502.

[6] R. L. Stewart and R. A. Russell, "A distributed feedback mechanism to regulate wall construction by a robotic swarm." Adaptive Behaviour, vol. 14, no. 1, pp. 21-51, 2006.

[7] T. Soleymani, V. Trianni, M. Bonani, F. Mondada, and M. Dorigo, "Autonomous Construction with Compliant Building Material," in Proceeding of the 13th International Conference on Intelligent Autonomous Systems (IAS-13), 2014.

[8] N. Napp and R. Nagpal, "Robotic Construction of Arbitrary Shapes with Amorphous Materials," in Conf on Robotics and Automation (ICRA), 2014.

[9] J. Werfel, D. Ingber, and R. Nagpal, "Collective construction of environmentally-adaptive structures," 2007 IEEE/RSJ International Conference on Intelligent Robots and Systems, pp. 2345-2352, Oct. 2007.

[10] K. Nagatani, S. Kiribayashi, Y. Okada, K. Otake, K. Yoshida, S. Tadokoro, T. Nishimura, T. Yoshida, E. Koyanagi, M. Fukushima, and S. Kawatsuma, "Emergency response to the nuclear accident at the fukushima daiichi nuclear power plants using mobile rescue robots." J. Field Robotics, vol. 30, no. 1, pp. 44-63, 2013.

[11] G. F. Knoll, Radiation detection and measurement, 2nd ed. New York: JOHN WILEY \& SONS, 1989.

[12] E. Gambao, C. Balaguer, and F. Gebhart, "Radiation exposure modeling for apartment living spaces with multiple radioactive sources." Health Physics, no. 3, p. 379, 1998.

[13] R. Goutam and R. Khare, "A Comprehensive paper on mobile robot path planning using wave front approach," Int. J. Tech, vol. 1, no. 2, pp. 143-148, 2011.

[14] Y. Hu and S. Yang, "A knowledge based genetic algorithm for path planning of a mobile robot," in Proceedings of the 2004 IEEE international Conference on Robotics \& Automation, April 2004, pp. 4350-4355.

[15] H. Ardiny, S. Witwicki, and F. Mondada, "Autonomous Construction of Separated Artifacts by Mobile Robots using SLAM and Stigmergy," in Conference on Autonomous and Robotic Construction of Infrastructure, June 2015. 